A. Kuribayashi

Nagoya Math. J.

Vol. 64 (1976), 97-115

\title{
ON THE GENERALIZED TEICHMÜLLER SPACES AND DIFFERENTIAL EQUATIONS
}

\author{
AKIKAZU KURIBAYASHI
}

It is well known that for the family $F$ of Riemann surfaces $\{R(z)\}$ defined by the equations $y^{2}=x(x-1)(x-z), z \in C-\{0,1\}$, we have one independent abelian differential $\omega=y^{-1} d x$ on each $R(z)$ and if we consider $z$ as a parameter on $C-\{0,1\}$, the integrals $\int_{g}^{h} y^{-1} d x(g, h=0,1, \infty)$ are solutions of the Gauss's differential equation

$$
z(z-1) \frac{d^{2} w}{d z^{2}}+(2 z-1) \frac{d w}{d z}+\frac{w}{4}=0
$$

If we take two suitable solutions $w_{1}(z), w_{2}(z)$ of the equation, and denote the ratios of $w_{1}(z)$ and $w_{2}(z)$ by $\tau$, then the inverse function $z(\tau)$ is a single valued holomorphic function on the upper-half $\tau$ plane.

The first aim of this paper is to consider families of Riemann surfaces for the differential equations of Fuchsian type, just as we have considered the family $F$ for the Gauss's differential equation. These are investigated in $\S 1$ and $\S 2$. The second aim is to investigate the analytic properties in these families by considering the symmetric domain $H$ which was introduced by Shimura [9] and the generalized Teichmüller space which was constructed in [5]. These are studied in $\S 3$. The main result here is Theorem (3.3.7), which is an answer to the extension of the case of the Gauss's differential equation.

Just as for the family $F$ the parameter $z$ is the Lambda function in the upper half plane, and is represented by the quotients of theta constants, our third aim is to similarly investigate the analyticity of the parameters, which we have in 1.3 , in the complex structure. We study this problem in $\S 4$, wherein an answer is given by Theorem (4.2.11).

Received September 19, 1975.

Revised October 23, 1975. 


\section{§1. A family $\Omega\left(g^{\prime}, n,\left\{\nu_{1}, \cdots, \nu_{r}\right\}\right)$ of Riemann surfaces}

1.1. Let $R$ be a compact Riemann surface of genus $g(\geq 1)$. Let $\sigma$ be an automorphism of $R$. We say that $(R, \sigma)$ and $\left(R^{\prime}, \sigma^{\prime}\right)$ are isomorphic if there exists a holomorphic bijection $f: R \rightarrow R^{\prime}$ such that $f \sigma=\sigma^{\prime} f$. We denote by $\langle R, \sigma\rangle$ the isomorphism class of $(R, \sigma)$. Let $n$ be a prime number and $\left\{\nu_{1}, \cdots, \nu_{r}\right\}$ be a set of positive integers such that $1 \leq \nu_{i}<n$ $(1 \leq i \leq r)$. We denote by $\Omega\left(g^{\prime}, n,\left\{\nu_{1}, \cdots, \nu_{r}\right\}\right)$ the set of all isomorphism classes of $(R, \sigma)$ satisfying the following conditions:

(1.1.1) (i) $\sigma$ is an automorphism of order $n$ with $r$ fixed points.

(ii) $R / G=R^{\prime}$ is of genus $g^{\prime}$, where $G$ is the cyclic group generated by $\sigma$.

(iii) $\sigma$ can be represented as $t_{i} \rightarrow \zeta^{\nu} t_{i}+\cdots(\zeta=\exp (2 \pi i / n))$, where $t_{i}$ is a local parameter at $q_{i}$, a fixed point of $\sigma$, and $i$ runs from 1 to $r$.

Clearly in (iii) the coefficient $\zeta^{\nu_{i}}$ does not depend on the choice of local parameter.

1.2. Let $K$ (resp. $K^{\prime}$ ) be the field of meromorphic functions on $R$ (resp. $R^{\prime}$ ). Then $K$ is a Galois extension of $K^{\prime}$, whose Galois group can be identified with $G$. Then there exists an element $y$ of $K$ such that $\sigma(y)=\zeta y, K=K^{\prime}(y)$ and $y^{n} \in K^{\prime}$. If $K^{\prime}$ is the rational function field, then we can write the equation of $R$ in the form

$$
\begin{aligned}
& y^{n}=\left(x-a_{0}\right)^{m_{0}}\left(x-a_{1}\right)^{m_{1}} \cdots\left(x-a_{s+1}\right)^{m_{s+1}}, n \nmid m_{0}+\cdots+m_{s+1}, \\
& 0<m_{i}<n(1 \leqq i \leqq s+1) .
\end{aligned}
$$

with distinct complex numbers $a_{0}, \cdots, a_{s+1}$. Here $r=s+3$, and we have $m_{i} \nu_{i} \equiv 1(\bmod n)$ for $0 \leq i \leq s+1$. Further we have a relation $2 g=(n-1)(s+1)$ and it is easy to see that we have $\left(\sum_{i=0}^{s+1} m_{i}\right) \nu_{r}+1 \equiv 0$ $(\bmod n)$.

In the equivalence class of $\langle R, \sigma\rangle$, we can find a representative $(R, \sigma)$ such that the equation of $R$ is

$$
y^{n}=x^{m_{0}}\left(x-z_{1}\right)^{m_{1}} \cdots\left(x-z_{s}\right)^{m_{s}}(x-1)^{m_{s+1}} .
$$

Here, $z_{1}, \cdots, z_{s}, 0$ and 1 are distinct complex numbers. We call this form of the equation a normal one and denote the Riemann surface defined by this equation by $R(z)$ and the complex vector space of all 
abelian differentials of $R(z)$ by $V(z)$, then we have the following lemma. (1.2.3) LEMma. $V(z)$ is spanned by abelian differentials of the form

(1) $\omega=x^{k_{0}}\left(x-z_{1}\right)^{k_{1}} \cdots\left(x-z_{s}\right)^{k_{s}}(x-1)^{k_{s+1}} y^{-\ell} d x$

$$
\left(0<\ell \leq n-1,0 \leq k_{0}, \cdots, k_{s+1}<n\right) \text {. }
$$

Here we must have

(2) $(n-1)+n k_{i}-\ell m_{i} \geq 0 \quad(0 \leq i \leq s+1)$,

(3) $\ell\left(m_{0}+\cdots+m_{s+1}\right)-n\left(k_{0}+\cdots+k_{s+1}\right) \geq n+1$.

Proof. $R(z)$ has an automorphism $\sigma: x \rightarrow x, y \rightarrow \zeta y$, where $\zeta$ is equal to $\exp (2 \pi i / n)$. Fixing a suitable basis of $V(z)$, we can represent $\sigma$ by a diagonal matrix as follows:

$$
\left(\begin{array}{c}
\omega_{1} \\
\vdots \\
\omega_{g}
\end{array}\right) \sigma=\left(\begin{array}{lll}
\zeta_{1} & & 0 \\
& \ddots & \\
0 & & \zeta_{g}
\end{array}\right)\left(\begin{array}{c}
\omega_{1} \\
\vdots \\
\omega_{g}
\end{array}\right) .
$$

Here $\zeta_{k}(1 \leq k \leq g)$ is a $n$-th root of unity. Hence, we have $\omega_{k} \sigma_{k}=\zeta_{k} \omega_{k}$ $(1 \leq k \leq g)$. It is well-known that $\omega_{k}$ can be expressed by a polynomial $\phi(x, y)$ in $x, y$ as follows:

$$
\omega_{k}=\phi(x, y) y^{-(n-1)} d x
$$

Therefore, we obtain $\omega_{k} \sigma=\zeta \phi(x, \zeta y) y^{-(n-1)} d x$. On the other hand, this is equal to $\zeta_{k} \omega_{k}=\zeta_{k} \phi(x, y) y^{-(n-1)} d x$. Hence we have $\phi(x, \zeta y)=\zeta^{\alpha} \phi(x, y)$ with an integer $\alpha(0 \leq \alpha \leq n-1)$. If we put

$$
\phi(x, y)=a_{0}(x) y^{m}+a_{1}(x) y^{n-1}+\cdots+a_{m}(x)
$$

with polynomials in $x, a_{0}(x), a_{1}(x), \cdots, a_{m}(x)$, then obviously we have

$$
\phi(x, \zeta y)=\sum_{i=0}^{m} a_{i}(x) \zeta^{m-i} y^{m-i}, \quad \zeta^{\alpha} \phi(x, y)=\sum_{i=0}^{m} a_{i}(x) \zeta^{\alpha} y^{m-i} .
$$

By the condition $\phi(x, \zeta y)=\zeta^{\alpha} \phi(x, y)$, every term except $a_{m-\alpha}(x) \zeta^{\alpha} y^{\alpha}$ must be zero. Consequently, the differentials $\omega_{k}(1 \leq k \leq g)$ which form a basis of $V(z)$ are of the form $\omega_{k}=f(x) y^{-\ell} d x$, where $\ell$ is a number $1 \leq \ell$ $<n-1$ and $f(x)$ is a polynomial in $x$. Our next problem is to see this polynomial is of the form $x^{k_{0}}\left(x-z_{1}\right)^{k_{1}} \cdots\left(x-z_{s}\right)^{k_{s}}(x-1)^{k_{s+1}}$ with some integers $k_{1}, \cdots, k_{s+1}$. Let these points of $R(z)$ which are over $0,1, z_{1}$, 
$\cdots, z_{s}, \infty$ be $q_{0}, q_{1}, q_{z_{1}}, \cdots, q_{z_{s}}, q_{\infty}$ respectively. Then we have

$$
\begin{aligned}
& \operatorname{div}(x)=n q_{0}-n q_{\infty}, \quad \operatorname{div}(x-1)=n q_{1}-n q_{\infty}, \\
& \operatorname{div}\left(x-z_{i}\right)=n q_{z_{i}}-n q_{\infty} \quad(1 \leq i \leq s), \\
& \operatorname{div}(d x)=(n-1) q_{0}+\sum_{i=1}^{s}(n-1) q_{z_{i}}+(n-1) q_{1}-(n-1) q_{\infty}, \\
& \operatorname{div}\left(y^{-\ell} d x\right)=\left\{(n-1)-\ell m_{0}\right\} q_{0}+\sum_{i=1}^{s}\left\{(n-1)-\ell m_{i}\right\} q_{z_{i}} \\
& \quad+\left\{(n-1)-\ell m_{s+1}\right\} q_{1}+\left\{\ell\left(\sum_{j=0}^{s+1} m_{j}\right)-(n-1)\right\} q_{\infty} .
\end{aligned}
$$

Hence we have $\ell\left(\sum_{j=0}^{s+1} m_{j}\right) \geq(n+1)$. First, we assume that $n-1-$ $\ell m_{i} \geq 0$ for all $i(0 \leq i \leq s+1)$. Then obviously we have $\ell\left(\sum_{j=0}^{s+1} m_{j}\right)-$ $(n+1)-t n \geq 0$. Here $t=\operatorname{deg} f(x)$. Consequently, $y^{-\ell} d x, \cdots, y^{-\ell} x^{t} d x$ are differentials of the first kind and $f(x) y^{-\ell} d x$ is generated by our differentials. Second, we assume that one of $(n-1)-\ell m_{i}(0 \leq i \leq s+1)$ is negative, say, $(n-1)-\ell m_{1}<0$. Then there exists an integer $k_{1} \leq t$ such that $(n-1)+n k_{1}-\ell m_{1} \geq 0$, and $f(x)$ must contain a factor $\left(x-z_{1}\right)^{k_{1}}$. Therefore we can write $f(x)=\left(x-z_{1}\right)^{k_{1}} g(x)$, where $g(x)$ is a polynomial. If we put $k_{0}=\operatorname{deg} g(x)$, then as before, $\left(x-z_{1}\right)^{k_{1}} y^{-\ell} d x, \cdots$, $x^{k_{0}}\left(x-z_{1}\right)^{k_{1}} y^{-\ell} d x$ are differentials of the first kind and again $f(x) y^{-\ell} d x$ is generated by our differentials. If two of $(n-1)-\ell m_{i}(0 \leq i \leq s+1)$ are negative, it goes similarly, and so on. Consequently, we can conclude that $V(z)$ is generated by differentials of the first kind of the form (1).

It is easy to see that the abelian differentials $\omega$ must satisfy the relations (2) and (3).

1.3. Now, we give a brief account of the generalized Teichmüller space $\Lambda$. We fix a couple $\left(R_{0}, \sigma_{0}\right)$ such that $\langle R, \sigma\rangle$ belongs to $\Omega\left(g^{\prime}, n\right.$, $\left.\left\{\nu_{1}, \cdots, \nu_{r}\right\}\right)$ and denote by $\Gamma\left(R_{0}, \sigma_{0}\right)$ the set of all the elements $\langle R, \sigma\rangle$ of $\Omega\left(g^{\prime}, n,\left\{\nu_{i}\right\}\right)$ such that $(R, \sigma)$ is topologically equivalent to $\left(R_{0}, \sigma_{0}\right)$, i.e., there exists a topological mapping $f: R_{0} \rightarrow R$ such that $f \sigma_{0}=\sigma f$. We consider a triple $(R, \sigma, \alpha)$ formed by a couple $(R, \sigma)$ such that $\langle R, \sigma\rangle$ of $\Gamma\left(R_{0}, \sigma_{0}\right)$ and a homotopy class $\alpha$ of orientation preserving topological mappings of $\left(R_{0}, \sigma_{0}\right)$ onto $(R, \sigma)$. We say that $(R, \sigma, \alpha)$ is isomorphic to $\left(R^{\prime}, \sigma^{\prime}, \alpha^{\prime}\right)$ if there exists an isomorphism of $(R, \sigma)$ onto $\left(R^{\prime}, \sigma^{\prime}\right)$ which belongs to the homotopy class $\alpha^{\prime} \alpha^{-1}$. We denote by $\langle R, \sigma, \alpha\rangle$ the isomorphism class of $(R, \sigma, \alpha)$ and the set of all classes $\langle R, \sigma, \alpha\rangle$ is denoted by $\Lambda\left(g^{\prime}, n,\left\{\nu_{i}\right\} ;\left(R_{0}, \sigma_{0}\right)\right)$ or $\Lambda\left(R_{0}, \sigma_{0}\right)$. We have the following [5]: 
(1.3.1) Lemma. The generalized Teichmüller space $\Lambda\left(g^{\prime}, n,\left\{\nu_{1}, \cdots, \nu_{r}\right\}\right.$; $\left.\left(R_{0}, \sigma_{0}\right)\right)$ is a simply connected $3 g^{\prime}-3+r$ dimensional complex analytic manifold. The mapping \& of $\Lambda\left(R_{0}, \sigma_{0}\right)$ into the ordinary Teichmüller space $T_{g}$, defined by corresponding $\langle R, \sigma, \alpha\rangle$ to $\langle R, \alpha\rangle$ is isomorphic.

If $g^{\prime}=0$, then each member of $\Omega\left(g^{\prime}, n,\left\{\nu_{i}\right\}\right)$ belongs to one $\Gamma\left(R_{0}, \sigma_{0}\right)$, and is of form $\langle R, \sigma\rangle$ where $R$ is given by the equation (1.2.2) [5].

Now, we denote one of the normal form of $\left(R_{0}, \sigma_{0}\right)$ by

$$
y^{n}=x^{m_{0}}\left(x-z_{1}^{(0)}\right)^{m_{1}} \cdots\left(x-z_{s}^{(0)}\right)^{m_{s}}(x-1)^{m_{s+1}}, n \nmid m_{0}+\cdots+m_{s+1} .
$$

Let $\lambda_{0}$ be $\left\langle R_{0}, \sigma_{0}, \alpha_{0}\right\rangle$ and let $\lambda$ be an arbitrary element of $\Lambda\left(R_{0}, \sigma_{0}\right)$. In the homotopy class $\alpha \alpha_{0}^{-1}$ there exists one and only one extremal quasi-conformal mapping $f: \lambda_{0} \rightarrow \lambda$ which has the property $f \sigma_{0}=\sigma f$ [5]. Then $f$ can be considered as a mapping of a Riemann sphere to a Riemann sphere. Put $f(0)=0, f(1)=1, f(\infty)=\infty$ and $f\left(z_{i}^{(0)}\right)=z_{i}(1 \leq i \leq s)$. We have as the equation of $\lambda$

$$
y^{n}=x^{m_{0}}\left(x-z_{1}\right)^{m_{1}} \cdots\left(x-z_{s}\right)^{m_{s}}(x-1)^{m_{s+1}}, n \nmid m_{0}+\cdots+m_{s+1},
$$

with these $0, z_{1}, \cdots, z_{s}$ and 1 . Obviously they are distinct from each other, and the parameters $z_{i}(1 \leq i \leq s)$ can be considered as functions on the generalized Teichmüller space $\Lambda\left(R_{0}, \sigma_{0}\right)$. Then we have the following lemma.

(1.3.4) Lemma. $z_{i}(1 \leq i \leq s)$ are continuous on $\Lambda\left(R_{0}, \sigma_{0}\right)$.

Proof. We have topologized $\Lambda\left(R_{0}, \sigma_{0}\right)$ by the Teichmüller metric

(1.3.5) $\operatorname{dist}\left(\lambda, \lambda^{\prime}\right)=\log K, \quad k=(K-1) /(K+1)$.

Here $K$ is the maximal dilatation of the extremal quasi-conformal mapping $g$ of $\lambda$ to $\lambda^{\prime}$. Since $g \in \alpha^{\prime} \alpha^{-1}$ commutes with the automorphism, it can be considered as a mapping of the Riemann sphere which fixes 0,1 and $\infty$, and which maps $z_{i}$ to $z_{i}^{\prime}(1 \leq i \leq s)$ in their equations. Then we have the following lemma [1].

$$
[g(\zeta), \zeta] \leq C\|\mu\|_{\infty} .
$$

Here $\left[\zeta, \zeta^{\prime}\right]$ denotes the spherical distance of $\zeta$ and $\zeta^{\prime}$, and $\mu$ is a measurable function with $k=\|\mu\|_{\infty}$. $C$ is a constant which does not depend on $k$. In fact, $g$ is the unique $\mu$-conformal mapping which fixes 0,1 and $\infty$ of the Riemann sphere. (1.3.6) shows the continuity of the 
functions $z_{i}$.

\section{§ 2. Hypergeometric differential equations}

2.1. As we see in $\S 1$, a differential of the first kind on a Riemann surface $R(z)$ defined by

$$
y^{n}=x^{m_{1}}(x-1)^{m_{2}}(x-z)^{m_{3}}, \quad n \nmid m_{1}+m_{2}+m_{3}
$$

is of the form

$$
\omega=x^{k_{1}}(x-1)^{k_{2}}(x-z)^{k_{3}} y^{-\ell} d x \quad\left(0<\ell \leq n-1,0 \leq k_{1}, k_{2}, k_{3}<n\right) .
$$

Now, by considering $z$ as a variable we can connect the differential with a differential equation of Fuchsian type. If we put

$$
\begin{aligned}
& \alpha=-\left(k_{1}+k_{2}+k_{3}\right)+\ell\left(m_{1}+m_{2}+m_{3}\right) n^{-1}-1, \\
& \beta=-k_{3}+\ell m_{3} n^{-1}, \\
& \gamma=-\left(k_{1}+k_{3}\right)+\ell\left(m_{1}+m_{3}\right) n^{-1},
\end{aligned}
$$

then the relations (2) and (3) in (1.2.3) that the differential of the first kind must satisfy gives us following conditions:

$$
\begin{aligned}
& \text { (i) } \beta-\gamma+1 \geq n^{-1}, \quad \gamma-\alpha \geq n^{-1}, \quad 1-\beta \geq n^{-1}, \alpha \geq n^{-1} \text {, } \\
& \text { (ii) } \alpha, \beta, \alpha-\gamma, \beta-\gamma \text { are not integers. }
\end{aligned}
$$

With these $\alpha, \beta, \gamma$, we construct a differential equation

$$
z(z-1) \frac{d^{2} w}{d z^{2}}+[(\alpha+\beta+1) z-\gamma] \frac{d w}{d z}+\alpha \beta w=0
$$

Then the solution $w(z)$ is given by

$$
w(z)=\int_{g}^{h} x^{\beta-\gamma}(x-1)^{\gamma-\alpha-1}(x-z)^{-\beta} d x,
$$

where $g, h$ are any two of 0,1 and $\infty$ [7]. This solution is nothing but the integral

$$
\int_{g}^{h} x^{k_{1}}(x-1)^{k_{2}}(x-z)^{k_{3}} y^{-\ell} d x .
$$

Conversely, consider the differential equation (2.1.5) with conditions

$$
\begin{aligned}
& \text { (i) } \beta-\gamma+1>0, \gamma-\alpha>0,1-\beta>0, \alpha>0 \text {, } \\
& \text { (ii) } \alpha, \beta, \alpha-\gamma, \beta-\gamma \text { are not integers, }
\end{aligned}
$$


(iii) $\alpha=a n^{-1}, \beta=b n^{-1}, \gamma=c n^{-1}$; where $n$ is a prime number and $a, b, c$ are integers.

Using Gaussian symbols we put

(2.1.9) $\quad k_{1}=[\beta-\gamma]+1, \quad k_{2}=[\gamma-\alpha-1]+1, \quad k_{3}=[-\beta]+1$

Choosing suitable $t_{i}$ such that $0<t_{i} n^{-1}<1$, we have $k_{1}-(\beta-\gamma)=t_{1} n^{-1}$, $k_{2}-(\gamma-\alpha-1)=t_{2} n^{-1}, k_{3}-(-\beta)=t_{3} n^{-1}$. Let $\ell$ be a maximal common divisor of $t_{1}, t_{2}$ and $t_{3}$. Then we have

$$
\begin{aligned}
& k_{1}-(\beta-\gamma)=\ell m_{1} n^{-1}, \quad k_{2}-(\gamma-\alpha-1)=\ell m_{2} n^{-1}, \\
& k_{3}-(-\beta)=\ell m_{3} n^{-1}
\end{aligned}
$$

with integers $m_{i}$ such that $1 \leq m_{i}<n(1 \leq i \leq 3)$. Hence we have $\ell\left(m_{1}+m_{2}+m_{3}\right) n^{-1}=k_{1}+k_{2}+k_{3}+1+\alpha$ and so we have $n \nmid\left(m_{1}+m_{2}+m_{3}\right)$. Thus we can construct with these $m_{1}, m_{2}, m_{3}$ and $n$ a Riemann surface (2.1.1). It is easy to see that $\omega=x^{k_{1}-\ell m_{1} n-1}(x-1)^{k_{2}-\ell m_{2} n-1}(x-z)^{k_{3}-\ell m_{3} n-1} d x$ is a differential of the first kind and $\int_{g}^{h} \omega$ is a solution of the given differential equation (2.1.5). Here $g, h$ are any two of $0,1, \infty$.

Now, consider a Riemann surface defined by the equation

$$
Y^{n^{\prime}}=X^{m_{1}^{\prime}}(X-1)^{m_{2}^{\prime}}(X-z)^{m_{3}^{\prime}}, \quad n^{\prime} \nmid m_{1}^{\prime}+m_{2}^{\prime}+m_{3}^{\prime},
$$

where $n^{\prime}$ is a prime integer and $m_{1}^{\prime}, m_{2}^{\prime}, m_{3}^{\prime}$ are integers less than $n^{\prime}$. We assume that

$$
\begin{gathered}
\omega^{\prime}=X^{k_{1}^{\prime}}(X-1)^{k_{2}^{\prime}}(X-z)^{k_{3}^{\prime}} Y^{-\ell^{\prime}} d x \\
\left(0<\ell^{\prime} \leq n^{\prime}-1,0 \leq k_{1}^{\prime}, k_{2}^{\prime}, k_{3}^{\prime}<n^{\prime}\right)
\end{gathered}
$$

is a differential of the first kind and further, assume that by considering $z$ as a variable the integral $\int_{g}^{h} \omega^{\prime}$ is a solution of the given differential equation (2.1.5). Here $g, h$ are any two of $0,1, \infty$. Then we have

$$
k_{1}^{\prime}-\ell^{\prime} m_{1}^{\prime} n^{\prime-1}=\beta-\gamma=k_{1}-\ell m_{1} n^{-1} .
$$

Hence we see that $n=n^{\prime}$. Also we see that $\ell^{\prime} m_{i}^{\prime}-\ell m_{i} \equiv 0(\bmod n)$ for all $i(1 \leq i \leq 3)$. There exists an integer $t^{\prime}$ such that $t^{\prime} \ell^{\prime} \equiv 1(\bmod n)$. Hence we have $m_{i}^{\prime}-t^{\prime} \ell m_{i} \equiv 0(\bmod n)$ for all $i(1 \leq i \leq 3)$. Put $t^{\prime} \ell=q$. Then the Riemann surface (2.1.11) is represented by 


$$
\begin{aligned}
Y^{n} & =X^{m_{1}^{\prime}}(X-1)^{m_{2}^{\prime}}(X-z)^{m_{3}^{\prime}} \\
& =\left\{X^{q m_{1}}(X-1)^{q m_{2}}(X-z)^{q m_{s}}\right\}\left\{X^{n p_{1}}(X-1)^{n p_{2}}(X-z)^{n p_{s}}\right\}
\end{aligned}
$$

with integers $p_{1}, p_{2}$ and $p_{3}$. By the birational transformation $X=\xi, Y=$ $\eta \xi^{p_{1}}(\xi-1)^{p_{2}}(\xi-z)^{p_{3}}(2.1 .11)$ is transformed to

$$
\eta^{n}=\xi^{q m_{1}}(\xi-1)^{q m_{2}}(\xi-z)^{q m_{3}} .
$$

However this is transformed to (2.1.1) by the birational transformation $\xi=x, \eta^{a^{\prime}} \xi^{m_{1} b^{\prime}}(\xi-1)^{m_{2} b^{\prime}}(\xi-z)^{m_{8} b^{\prime}}=y$. Here $a^{\prime}, b^{\prime}$ are integers such that $a^{\prime} q+b^{\prime} n=1$. Therefore, (2.1.1) and (2.1.11) are conformally equivalent. Thus, we obtain the following theorem:

(2.1.15) THEOREM. Consider a hypergeometric differential equation (2.1.5) with conditions (i), (ii) and (iii). Then there exists a family of Riemann surfaces $\{R(z)\}$ defined by (2.1.1) and by considering $z$ as a variable a differential $\omega$ of the first kind of $R(z)$ such that the integral $\int_{g}^{h} \omega(g, h=0,1, \infty)$ is a solution of (2.1.5). The Riemann surface $R(z)$ is uniquely determined up to conformal equivalence in usual sense. However if we consider in the family of Riemann surfaces $\Omega\left(g^{\prime}, n,\left\{\nu_{i}\right\}\right)$ it is uniquely determined.

2.2. Now, we assume that there exist positive integers $s_{1}, s_{2}$ and $s_{3}$, which satisfy the inequality $1 / s_{1}+1 / s_{2}+1 / s_{3}<1$ and the system of equalities

$$
\begin{aligned}
1-\beta & =\left(1 / s_{1}+1 / s_{2}-1 / s_{3}+1\right) / 2, \\
1+\beta-\gamma & =\left(1 / s_{3}+1 / s_{1}-1 / s_{2}+1\right) / 2, \\
\gamma-\alpha & =\left(1 / s_{2}+1 / s_{3}-1 / s_{1}+1\right) / 2,
\end{aligned}
$$

in the differential equation (2.1.5). For the meaning of this see [7].

We must study what becomes of the equation of a Riemann surface under the condition (2.2.1). (2.2.1) says there exist integers $s_{1}, s_{2}, s_{3}$ such that

$$
\begin{aligned}
& k_{3}-\ell m_{3} n^{-1}=\left(1 / s_{1}+1 / s_{2}-1 / s_{3}-1\right) / 2 \\
& k_{1}-\ell m_{1} n^{-1}=\left(1 / s_{3}+1 / s_{1}-1 / s_{2}-1\right) / 2 \\
& k_{2}-\ell m_{2} n^{-1}=\left(1 / s_{2}+1 / s_{3}-1 / s_{1}-1\right) / 2 .
\end{aligned}
$$

Since $n$ is prime, by a simple calculation we see that $k_{1}=k_{2}=k_{3}$ and 
$m_{1}=m_{2}=m_{3}$. Here we must notice that $s_{1}=s_{2}=s_{3}$ and the Riemann surface defined by $Y^{n}=X^{m}(X-1)^{m}(X-z)^{m}$ is conformally equivalent to the Riemann surface defined by $y^{n}=x(x-1)(x-z)$. Indeed, we need only to do a birational transformation such that $x=X, y=Y^{a} X^{b}$ $\cdot(X-1)^{b}(X-z)^{b}$. It is easily seen that we can have the same differential equation from these Riemann surfaces. Here, $a$ and $b$ are integers such that $a m+b n=1$.

2.3. As we see in $\S 1$, a differential of the first kind on a Riemann surface $R(z)$ defined by

$$
y^{n}=x^{m_{0}}\left(x-z_{1}\right)^{m_{1}} \cdots\left(x-z_{s}\right)^{m_{s}}(x-1)^{m_{s+1}}, \quad n \nmid m_{0}+\cdots+m_{s+1},
$$

is of the form

$$
\begin{array}{r}
\omega=x^{k_{0}}\left(x-z_{1}\right)^{k_{1}} \cdots\left(x-z_{s}\right)^{k_{s}}(x-1)^{k_{s+1}} y^{-\ell} d x \\
\left(0<\ell \leq n-1,0 \leq k_{i}<n\right) .
\end{array}
$$

Now, by considering $z_{1}, \cdots, z_{s}$ as independent variables we can connect the differential with a partial differential equation of Appellian type by the same method which we used in 2.1 and 2.2. Namely, if we put

$$
\begin{aligned}
& \alpha=-\sum_{i=0}^{s+1} k_{i}+\ell\left(\sum_{i=0}^{s+1} m_{i}\right) n^{-1}-1, \\
& \beta_{i}=-k_{i}+\ell m_{i} n^{-1} \quad(1 \leq i \leq s), \\
& \gamma=-\sum_{i=0}^{s} k_{i}+\ell\left(\sum_{i=0}^{s} m_{i}\right) n^{-1},
\end{aligned}
$$

then we obtain the following conditions by the relations (2) and (3) in (1.2.3):

$$
\begin{aligned}
& \text { (i) } \sum_{i=1}^{s} \beta_{i}-\gamma+1 \geq n^{-1}, \gamma-\alpha \geq n^{-1}, 1-\beta_{i} \geq n^{-1}(1 \leq i \leq s), \\
& \alpha \geq n^{-1},
\end{aligned}
$$

(ii) $\alpha, \beta_{i}(1 \leq i \leq s), \gamma-\alpha, \gamma-\sum_{i=1}^{s} \beta_{i}$ are not integers.

With these $\alpha, \beta_{i}(1 \leq i \leq s)$ and $\gamma$ we construct a partial differential equation as follows [2: pp. 117-120]:

$$
\begin{array}{ll}
A_{i} \frac{\partial^{2} w}{\partial z_{i}^{2}}+\sum_{j=1}^{s} B_{j}^{(i)} \frac{\partial w}{\partial z_{j}}+C_{i} w=0 & (1 \leq i \leq s) \\
\left(z_{i}-z_{j}\right) \frac{\partial^{2} w}{\partial z_{i} \partial z_{j}}-\beta_{j} \frac{\partial w}{\partial z_{i}}+\beta_{i} \frac{\partial w}{\partial z_{j}}=0 & (i \neq j) .
\end{array}
$$


Here, $A_{i}=z_{i}\left(z_{i}-1\right), C_{i}=\alpha \beta_{i}$ and

$$
\begin{aligned}
B_{j}^{(i)}= & -\beta_{i} \frac{z_{j}\left(z_{j}-1\right)}{z_{i}-z_{j}} \quad(i \neq j) \\
B_{i}^{(i)}= & z_{i}\left(z_{i}-1\right) \sum_{\substack{1 \leq j \leq s \\
j \neq i}} \frac{\beta_{i}}{z_{i}-z_{j}}-\left(\gamma-\sum_{i=1}^{s} \beta_{i}\right)-\beta_{i} \\
& +\left[\alpha-\left(\sum_{i=1}^{s} \beta_{i}\right)+2 \beta_{i}+1\right] z_{i} .
\end{aligned}
$$

Then a solution $w\left(z_{1}, \cdots, z_{s}\right)$ is given by the integral

$$
w\left(z_{1}, \cdots, z_{s}\right)=\int_{g}^{h} x^{\beta_{1}+\cdots+\beta_{s}-\gamma}\left(x-z_{1}\right)^{-\beta_{1}} \cdots\left(x-z_{s}\right)^{-\beta_{s}}(x-1)^{\gamma-\alpha-1} d x,
$$

where $g, h$ are any two of $0,1, z_{1}, \cdots, z_{s}$ and $\infty$.

Conversely, we consider the partial differential equation of Appellian type (2.3.5) with conditions

(i) $\sum_{i=1}^{s} \beta_{i}-\gamma+1>0, \gamma-\alpha>0,1-\beta_{i}>0(1 \leq i \leq s), \alpha>0$,

(ii) $\alpha, \beta_{i}(1 \leq i \leq s), \gamma-\alpha$ and $\gamma-\sum_{i=1}^{s} \beta_{i}$ are not integers,

(iii) $\alpha=a n^{-1}, \beta_{i}=b_{i} n^{-1}, \gamma=c n^{-1}$; where $n$ is a prime number and $a, b_{i}(1 \leq i \leq s), c$ are integers.

Then we have $k_{0}, \cdots, k_{s+1}$ and $m_{0}, \cdots, m_{s+1}$ such that $n \nmid m_{0}+\cdots+m_{s+1}$. Thus, we can construct a family of Riemann surfaces $\{R(z)\}, y^{n}=x^{m_{0}}$ $\cdot\left(x-z_{1}\right)^{m_{1}} \cdots\left(x-z_{s}\right)^{m_{s}}(x-1)^{m_{s+1}}$, with these $m_{i}(0 \leq i \leq s+1)$ and $n$. Also we can construct a differential $\omega$ of the first kind on $R(z)$ such that the integral $\int_{g}^{h} \omega$ is a solution of the given differential equation (2.3.5). $R(z)$ is uniquely determined up to conformal equivalence in the usual sense.

\section{§3. Periods of Riemann surfaces}

3.1. We recall some results of Shimura $[9,10]$. Let $\boldsymbol{Q}$ be the rational number field and let $K=\boldsymbol{Q}(\zeta), \zeta=\exp (2 \pi i / n)$. Here we assume that $n$ is a prime number. Clearly $[K: Q]=n-1$. Put $2 h=n-1$. We denote by $\rho$ the complex conjugation. Let $\Phi$ be a representation of $K$ by complex matrix of size $g$. We say that a triple $\mathscr{P}=(A, C, \theta)$ is a polarized abelian variety of type $(K, \Phi, \rho)$ if the following conditions are satisfied: 
(3.1.1) (i) $A$ is an Abelian variety of dimension $g$, defined over the complex number field $C$.

(ii) $\theta$ is an isomorphism of $K$ into $\operatorname{End}_{Q}(A)$, and the representation of $\theta(x)$ for $x \in K$ by an analytic coordinate system of $A$ is equivalent to $\Phi(x)$.

(iii) $C$ is a polarization of $A$, and the involution of $\operatorname{End}_{Q}(A)$ determined by $C$ coincides with $\theta(x) \rightarrow \theta\left(x^{\rho}\right)$ on $\theta(K)$.

Let $\mathscr{P}=(A, C, \theta)$ be of type $(K, \Phi, \rho)$. Take a complex torus $C^{g} / D$ isomorphic to $A$, where $D$ is a lattice in $C^{g}$. We may choose the coordinate system of $C^{g}$ so that $\theta(a)$ is represented by the matrix $\Phi(a)$ for every $a \in K$. Then we can find $u=g / h$ vectors $\mathfrak{x}_{1}, \cdots, \mathfrak{x}_{u}$ such that $\boldsymbol{Q} D=K \mathfrak{x}_{1}+\cdots+K \mathfrak{x}_{u} . \quad$ For every $\boldsymbol{a}=\left(a_{1}, \cdots, a_{u}\right) \in K^{u}$, put $\mathfrak{x}(\boldsymbol{a})=\Phi\left(a_{1}\right) \mathfrak{x}_{1}$ $+\cdots+\Phi\left(a_{u}\right) \mathfrak{x}_{u}$. Then, the mapping $a \rightarrow \mathfrak{x}(a)$ is an isomorphism of $K^{u}$ onto $Q D$. Let $M$ be the inverse image of $D$ by this mapping. Let $E(x, \mathfrak{y})$ be a non-degenerate Riemann form on $C^{g} / D$ corresponding to a basic polar divisor in $C$. There exists an element $t_{i j} \in K$ such that $E\left(\Phi(a) \mathfrak{c}_{i}, \mathfrak{r}_{j}\right)=\operatorname{tr}\left(a t_{i j}\right)(1 \leq i, j \leq s+1)$. Put $T=\left(t_{i j}\right)$. Let $\sigma_{1}, \cdots \sigma_{h}, \sigma_{1} \rho$, $\cdots, \sigma_{h} \rho$ be all the isomorphisms of $K$ into $C$, and let $r_{\mu}$ (resp. $s_{\mu}$ ) be the multiplicity of $\sigma_{\mu}\left(\operatorname{resp} . \sigma_{\mu} \rho\right.$ ) in $\Phi$. In order to ensure the existence of $\mathscr{P}$ of type $(K, \Phi, \rho)$, the following relation should be satisfied: $2 g=$ $(n-1)\left(r_{\mu}+s_{\mu}\right)(1 \leq \mu \leq h)$. Let $H_{\mu}$ be the space of all complex matrices $Z_{\mu}$ with $r_{\mu}$ rows and $s_{\mu}$ columns such that $1-Z_{\mu}{ }^{t} Z_{\mu}$ is positive definite, and let $H=H_{1} \times \cdots \times H_{h}$. The dimension of $H$ is equal to $\sum_{\mu=1}^{h} r_{\mu} s_{\mu}$. If we fix $T$ and $M$, then we get an analytic family of polarized abelian varieties of type $(K, \Phi, \rho)$ parametrized by the points of $H$. We denote by $\sum$ this family and $\mathscr{P}_{z}$ the member of $\sum$ determined by $z \in H$. There exists a discontinuous group $G$ of transformations on $H$, and two members $\mathscr{P}_{z}$ and $\mathscr{P}_{z^{\prime}}$ of $\sum$ are isomorphic if and only if $z=U\left(z^{\prime}\right)$ for an element $U$ of $G$. Therefore, the isomorphism classes of members of $\sum$ are in one to one correspondence with the points of the quotient space $H / G$.

3.2. Now, let $\Omega\left(g^{\prime}, n,\left\{\nu_{i}\right\}\right)$ be the family of Riemann surfaces defined in 1.1. If $g^{\prime}=0$, each member of $\Omega\left(g^{\prime}, n,\left\{\nu_{i}\right\}\right)$ is of the form $\langle R, \sigma\rangle$ where $R$ is given by the equation (1.2.2). In this case, using all the member of $\Omega\left(g^{\prime}, n,\left\{\nu_{i}\right\}\right)$ we can construct the generalized Teichmüller space. Moreover, a basis of the vector space $V$ of $R(z)$ is given by Lemma 
(1.2.3). Therefore, the representation $\Phi(\sigma)$ of $\sigma$ is given by

$$
\Phi(\sigma)=\left(\begin{array}{ccc}
\zeta^{\alpha_{1}} & & 0 \\
& \ddots & \\
0 & & \zeta^{\alpha_{\sigma}}
\end{array}\right) .
$$

Here, $g$ is the genus of $R$ and $\alpha_{i} \geq 1(1 \leq i \leq g)$. $\Phi(\sigma)$ can be identified with the representation $\Phi(\zeta)$ of $K=Q(\zeta)$ which we considered in 3.1.

Let the first homology group with coefficients in $\boldsymbol{Q}$ of $R$ be $H_{1}(R, Q)$, and let the endomorphism of $H_{1}(R, Q)$ induced naturally by the automorphism $\sigma$ of $R$ be $\Phi_{*}(\sigma)$. Define $\Phi_{*}(\alpha)$ by

$$
\Phi_{*}(\alpha)=a_{0}+a_{1} \Phi_{*}(\sigma)+\cdots+a_{n-2} \Phi_{*}(\sigma)^{n-2}
$$

where, $a=a_{0}+a_{1} \zeta+\cdots+a_{n-2} \zeta^{n-2},\left(a_{0}, a_{1}, \cdots, a_{n-2} \in \boldsymbol{Q}\right)$. Then, $\Phi_{*}(a)$ operates on $H_{1}(R, \boldsymbol{Q})$. Hence, $H_{1}(R, \boldsymbol{Q})$ can be regarded as a vector space over $\Phi_{*}(K)$. On the other hand, we have $2 g=(n-1)(s+1)$ by the formula of Riemann-Hurwitz. Therefore, there exists $s+1$ vectors $Z_{1}$, $\cdots, Z_{s+1}$ such that

$$
H_{1}(R, \boldsymbol{Q})=\Phi_{*}(K) Z_{1}+\cdots+\Phi_{*}(K) Z_{s+1} .
$$

Now, it would be interesting to represent explicitly a basis of $H_{1}(R, Q)$ over $K$. Let $x_{0}$ be an arbitrary point on the $x$-sphere distinct from $0, z_{1}, \cdots, z_{s}, 1$ and $\infty$. We connect $x_{0}$ to these points by curves which have no intersection with each other except for $x_{0}$. Denote these curves by $\alpha_{0}, \cdots, \alpha_{s+1}, \alpha_{s+2}$ respectively. Fix a point $p_{0}=\left(x_{0}, y_{0}\right)$ on $R$ and denote by $\tilde{\alpha}_{i}$ the lift of $\alpha_{i}$ with the initial point at $p_{0}$. We put

$$
\begin{aligned}
& \text { (i) } C_{i}=\tilde{\alpha}_{i}+\sigma^{m_{i}} \tilde{\alpha}_{i}+\cdots+\sigma^{m_{i}\left(\nu_{i}-1\right)} \tilde{\alpha}_{i} \quad(0 \leq i \leq s+2) \text {, } \\
& \text { (ii) } Z_{j}=C_{j-1}-C_{j} \quad(1 \leq j \leq s+1) \text {. }
\end{aligned}
$$

Then we see easily that $C_{j}$ is a curve on $R$ connecting $p_{0}$ and $\left(x_{0}, \zeta y_{0}\right)$, and that $Z_{j}$ is a closed curve. Moreover we can prove $Z_{1}, \ldots, Z_{s+1}$ are a basis over $K=Q(\zeta)$ and $Z_{1}, \sigma Z_{1}, \cdots, \sigma^{n-2} Z_{1} ; \cdots ; Z_{s+1}, \sigma Z_{s+1}, \cdots, \sigma^{n-2} Z_{s+1}$ are a basis over the ring of integers $Z$.

3.3. Consider a point $z$ of $H$ which corresponds to $(R, \sigma)$ of $\Omega\left(g^{\prime}, n\right.$, $\left.\left\{\nu_{i}\right\}\right)$. We know that $\mathscr{P}_{z}$ is isomorphic to $\mathscr{P}_{z^{\prime}}$ if and only if $(R, \sigma)$ is isomorphic to $\left(R^{\prime}, \sigma^{\prime}\right)$, where $z^{\prime}$ corresponds to $\left(R^{\prime}, \sigma^{\prime}\right)$ of $\Omega\left(g^{\prime}, n,\left\{\nu_{i}\right\}\right)$, i.e., there exists an injection of $\Gamma\left(R_{0}, \sigma_{0}\right)$ into $H / G$. 
Now take a point $z=\left(z_{1}, \cdots, z_{s}\right)$ of $C^{s}$. Here we assume that $z_{1}$, $\cdots, z_{s}, 0$ and 1 are different from each other. We denote by $\dot{C}^{s}$ the set which consists of such points. Let $z=\left(z_{1}, \cdots, z_{s}\right)$ be an arbitrary point of $\dot{C}^{s}$. We can associate to $z$ a point $\langle R, \sigma\rangle$ of $\Gamma\left(R_{0}, \sigma_{0}\right)$ by the equation

$$
y^{n}=x^{m_{0}}\left(x-z_{1}\right)^{m_{1}} \cdots\left(x-z_{s}\right)^{m_{s}}(x-1)^{m_{s+1}}, \quad n \nmid m_{0}+\cdots+m_{s+1} .
$$

There exists an extremal quasi-conformal mapping of $\left\langle R_{0}, \sigma_{0}\right\rangle$ defined by (1.3.2) to $\langle R, \sigma\rangle$ defined by (3.3.1) [5]. Therefore there exists at least one $\lambda$ of $\Lambda\left(R_{0}, \sigma_{0}\right)$ such that the equation of $\lambda$ is (3.3.1), and we have a surjective map $z$ of $\Lambda\left(R_{0}, \sigma_{0}\right)$ to $\dot{C}^{s}$.

Take a point $z$ of $\dot{C}^{s}$. Let $z^{\prime}=\left(z_{1}^{\prime}, \cdots, z_{s}^{\prime}\right)$ be a point of $\dot{C}^{s}$ in a neighborhood of $z$. We denote by $R\left(z^{\prime}\right)$ the Riemann surface defined by (1.2.2) with this $z^{\prime}$. Then, we can take a basis of $V\left(z^{\prime}\right)$ of $R\left(z^{\prime}\right),\left\{\omega_{1}, \cdots\right.$, $\left.\omega_{g}\right\}$, each of which is a differential of the form (1.2.3). Consider $s+1$ vectors $\mathfrak{x}_{i}\left(z^{\prime}\right)(1 \leq i \leq s+1)$ which are represented by

$$
{ }^{t} \mathfrak{X}_{i}\left(z^{\prime}\right)=\left(\int_{z_{i}} \omega_{1}, \cdots, \int_{z_{i}} \omega_{g}\right) \text {. }
$$

Here $Z_{i}$ can be taken constant in the neighborhood of $z$. Therefore, $\mathfrak{x}_{i}\left(z^{\prime}\right)$ are holomorphic in the neighborhood. Moreover, we have by the same method as in [5] the following lemma.

(3.3.3) LEMma. Let the data corresponding to a point $z^{\prime}$ in a small neighborhood of $z$ be $\left\{\mathfrak{x}_{1}\left(z^{\prime}\right), \cdots, \mathfrak{x}_{s+1}\left(z^{\prime}\right) ; M\left(z^{\prime}\right), T\left(z^{\prime}\right)\right\}$. Then $M\left(z^{\prime}\right), T\left(z^{\prime}\right)$ are constants in the neighborhood.

Hence, we see that each coordinate of $\mathscr{P}_{z^{\prime}}$ is a holomorphic function in the neighborhood of $z$. We denote by $F\left(z^{\prime}\right)=\left(F_{1}\left(z^{\prime}\right), \cdots, F_{N}\left(z^{\prime}\right)\right)$ the coordinates of $\mathscr{P}_{z^{\prime}}$. Here $N$ is the dimension of the symmetric domain $H$ which corresponds to the generalized Teichmüller space $\Lambda\left(R_{0}, \sigma_{0}\right)$. Let $G$ be the discontinuous group mentioned in 3.1. Then, we have the following lemma [5].

(3.3.4) Lemma. There exists a holomorphic mapping $w(\lambda)$ of $\Lambda\left(R_{0}, \sigma_{0}\right)$ into $H$, which is a G-invariant mapping.

Now, we assume that for a point $\mathscr{P}_{z}$ of $H$ there exists a point $\lambda$ of $\Lambda$ which corresponds to $\mathscr{P}_{\beta^{\prime}}$. We denote the coordinates of $\mathscr{P}_{z^{\prime}}$ by $\left(z_{1}^{\prime}, \cdots\right.$, 
$\left.z_{N}^{\prime}\right)$. Then, $z_{1}^{\prime}, \cdots, z_{N}^{\prime}$ are holomorphic functions of $\lambda^{\prime}$ in a neighborhood of $\lambda$. Thus, we obtain the following relation:

$$
\jmath_{1}^{\prime}\left(\lambda^{\prime}\right)=F_{1}\left(z^{\prime}\right), \cdots, \jmath_{N}^{\prime}\left(\lambda^{\prime}\right)=F_{N}\left(z^{\prime}\right)
$$

in the neighborhood $U(\lambda)$ of $\lambda$ and the neighborhood $W(z)$ of $z$. Here we have $z^{\prime}\left(\lambda^{\prime}\right) \in W(z)$ for every $\lambda^{\prime} \in U(\lambda)$. We have the following diagram:

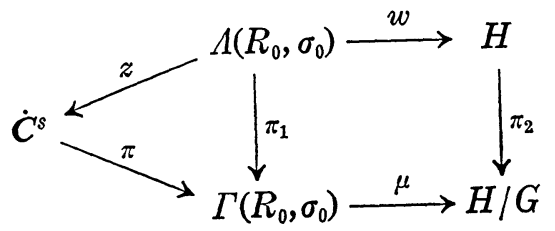

Here we give a complex structure in $\Gamma\left(R_{0}, \sigma_{0}\right)$ naturally by $\dot{C}^{s}$, and the mapping $\mu$ is a continuous injection [10]. Moreover, the mapping $\mu$ can be considered as follows. Take a point $\langle R, \sigma\rangle$ of $\Gamma\left(R_{0}, \sigma_{0}\right)$ and a small neighborhood of that point. Then the compositiom $\pi_{2} F \pi^{-1}$ is well defined in the neighborhood and coincides with $\mu$. Since $\Gamma\left(R_{0}, \sigma_{0}\right)$ is a normal analytic space and $\mu$ is continuous we see that $\mu$ is holomorphic by the theorem of Riemann [4]. Further, we remark that $z$ and $\pi_{1}(=\pi z)$ are continuous.

Now, take an arbitrary point $\lambda$ of $\Lambda\left(R_{0}, \sigma_{0}\right)$. Consider the point $\pi_{1}(\lambda)$ of $\Gamma\left(R_{0}, \sigma_{0}\right)$. Since $\mu$ is holomorphic injection there exist a neighborhood $W\left(\pi_{1}(\lambda)\right)$ of $\Gamma\left(R_{0}, \sigma_{0}\right)$ and a neighborhood $V$ of $\mu\left(\pi_{1}(\lambda)\right)$ in $H / G$ such that the restriction of $\mu$ to $W\left(\pi_{1}(\lambda)\right)$ is a proper mapping, i.e., $\mu\left(W\left(\pi_{1}(\lambda)\right)\right.$ is an analytic set in $V$ [8]. Let $A$ be the singular locus in $\mu\left(W\left(\pi_{1}(\lambda)\right)\right.$. Consider the set $w^{-1} \pi_{2}^{-1}(A)$ in $w^{-1} \pi_{2}^{-1}(V)$ which contains $\lambda$. In a suitable $U_{0}(\lambda)$, the dimension of $w^{-1} \pi_{2}^{-1}(A) \cap U_{0}(\lambda)$ is lower than the dimension of $U_{0}(\lambda)$. In fact, $\Lambda\left(R_{0}, \sigma_{0}\right)$ is a covering of $\Gamma\left(R_{0}, \sigma_{0}\right)$ and so $\pi_{2} w\left(U_{0}(\lambda)\right)$ cannot be contained in the set $A$.

By the way, $\pi_{1}$ is holomorphic in $U_{0}(\lambda)-w^{-1} \pi_{2}^{-1}(A)$ and continuous in $\Lambda\left(R_{0}, \sigma_{0}\right)$. Then by the theorem of Riemann, $\pi_{1}$ is holomorphic in $U_{0}(\lambda)$. Therefore, $\pi_{1}$ is a holomorphic covering of $\Lambda\left(R_{0}, \sigma_{0}\right)$ onto $\Gamma\left(R_{0}, \sigma_{0}\right)$. $\pi$ is also a holomorphic covering of $\dot{C}^{s}$ onto $\Gamma\left(R_{0}, \sigma_{0}\right)$, and $z$ is a continuous mapping of $\Lambda\left(R_{0}, \sigma_{0}\right)$ onto $\dot{C}^{s}$. By the same method as above we obtain the following theorem.

(3.3.7) THEOREM. The parameters $z_{1}, \cdots, z_{s}$ in (1.2.2) are single valued 
holomorphic functions on the generalized Teichmüller space $\Lambda\left(R_{0}, \sigma_{0}\right)$ which is constructed from $\Omega\left(g^{\prime}, n,\left\{\nu_{i}\right\}\right)$.

3.4. We should notice that the formula (3.3.5) is a generalization of the formula

$$
\tau=w_{1}(z) / w_{2}(z), \quad \operatorname{Im} \tau>0
$$

in the case of

$$
y^{2}=x(x-1)(x-z), \quad z \in C-\{0,1\} .
$$

Moreover, (3.3.7) is surely an extension of the function $z(\tau)$ on the upper half $\tau$-plane. In our case of (1.2.2), the space which corresponds to the upper half plane may be the symmetric domain $H$. However, (3.3.7) shows that it is not the symmetric domain $H$ but the generalized Teichmüller space which plays the role of the upper half plane.

It would be said that the formula (3.3.5) gives an answer to the so-called "Schottky Problem" in the special case.

In the present paper, we treated the symmetric domain $H$ which was constructed by Shimura. However, it goes quite similarly with the symmetric domain $H_{g}$ which was introduced by Siegel. Because the diagram analogous to (3.3.6) holds by the theorem of Torelli.

\section{§4. A representation of the parameters by Theta constants}

4.1. We give a brief account of the Theta function together with an explanation of notations. Let $R$ be a Riemann surface of genus $g$ and $A_{k}, B_{k}(1 \leq k \leq g)$ be a canonical dissection of $R$. Let $p_{0}$ be a common point of all $A_{k}, B_{k}(1 \leqq k \leqq g)$. The point $p_{0}$ is also the initial point for integration. Let $d w_{1}, \cdots, d w_{g}$ be a basis of the differentials of the first kind of $R$ and $d \mathfrak{w}$ be the vector $\left(d w_{1}, \cdots, d w_{g}\right)$. Let

$$
\begin{aligned}
w_{\ell}(p) & =\int_{p_{0}}^{p} d w_{\ell} \quad(1 \leqq \ell \leqq g), \\
\mathfrak{w}(p) & =\int_{p_{0}}^{p} d \mathfrak{w},
\end{aligned}
$$

where $p$ is the variable point on $R$ and the integration paths are to be selected on the canonically dissected Riemann surface $R^{*}$. We assume that with this basis the period matrix has the form $[E Z]$, where $E$ is the unit matrix of size $g$, and $Z=X+i Y$ satisfies the Riemann's rela- 
tion

$$
X={ }^{t} X, Y={ }^{t} Y \quad \text { and } \quad Y>0 \text {, }
$$

The Theta function formed with $Z$ is defined by

$$
\theta(\mathfrak{g})=\theta(\mathfrak{g}, Z)=\sum_{\mathfrak{n}} \exp \left(\pi i^{t} \mathfrak{n} Z \mathfrak{n}+2 \pi i^{t} \mathfrak{n} \mathfrak{g}\right)
$$

and $\theta(\mathfrak{g})$ satisfies the functional relation

$$
\theta(\mathfrak{g}+\mathfrak{g}+Z \mathfrak{h})=\theta(\mathfrak{g}) \exp \left(-i \pi^{t} \mathfrak{h} Z \mathfrak{h}-2 \pi i^{t} \mathfrak{h} \mathfrak{g}\right),
$$

where ${ }^{t} \mathfrak{g}=\left(g_{1}, \cdots, g_{g}\right),{ }^{t} \mathfrak{h}=\left(h_{1}, \cdots, h_{g}\right)$ are arbitrary integer vectors. We put $f(\boldsymbol{p})=\theta(\mathfrak{w}(\boldsymbol{p})-\mathfrak{g})$, where ${ }^{t_{\mathfrak{w}}}(\boldsymbol{p})=\left(w_{1}(\boldsymbol{p}), \cdots, w_{g}(\boldsymbol{p})\right)$ and ${ }^{t} \mathfrak{g}=\left(s_{1}, \cdots\right.$, $\left.s_{g}\right)$. With a circuit of $\boldsymbol{p}$ along a closed curve on $R, \mathfrak{w}(\boldsymbol{p})-\xi$ changed by a summand of the form $g+Z \mathfrak{h}$, and $f(\boldsymbol{p})$ is multiplied by the nonzero factor $\exp \left(-i \pi^{t} \mathfrak{h} Z \mathfrak{h}-2 \pi i^{t} \mathfrak{h}(\mathfrak{w}(\boldsymbol{p})-\mathfrak{g})\right)$. If for a fixed $\mathfrak{I}, f(\boldsymbol{p})$ does not vanish identically in $p$, then it has exactly $g$ zeros on $R$. Let $c$ be the vector consisting of the $g$ quantities

$$
c_{k}=\sum_{\ell=1}^{g} \int_{A \ell} w_{k}(\boldsymbol{p}) d w_{\ell}-(1 / 2) z_{k k} \quad(1 \leqq k \leqq g) .
$$

Now, if we choose $\mathfrak{g}$ such that the function $f(\boldsymbol{p})=\theta(\mathfrak{w}(\boldsymbol{p})-\mathfrak{g}+\mathfrak{c})$ does not vanish identically in $p$, then its $g$ zeros $q_{1}, \cdots, q_{g}$ satisfy

$$
\sum_{\ell=1}^{g} \mathfrak{w}\left(\boldsymbol{q}_{\ell}\right) \equiv \mathfrak{Z}
$$

It is known that $\theta(\mathfrak{w}(\boldsymbol{p})-\mathfrak{g}+\mathfrak{c})$ does not vanish identically in $p$, iff the inverse problem for the vector $\xi$ is uniquely determined, i.e., the equation (4.1.5) has a unique solution $q_{1}, \cdots, q_{g}$. In this case, the divisor $D=q_{1}+\cdots+q_{g}$ is general. We shall call an integral divisor $D=p_{1}$ $+\cdots+\boldsymbol{p}_{m}$ general if there are no non-constant meromorphic functions $f$ on $R$ with the property $\operatorname{div}(f)+D>0$.

4.2. Now, for our investigation the following [11] is useful.

(4.2.1) LEMMA. Let $f(\boldsymbol{p})$ be a meromorphic function on $R$ with the zeros $\boldsymbol{a}_{1}, \cdots, \boldsymbol{a}_{m}$ and the poles $\boldsymbol{b}_{1}, \cdots, \boldsymbol{b}_{m}$. We choose a general integral divisor $\boldsymbol{x}_{1}+\cdots+\boldsymbol{x}_{g-1}$ of degree $g-1$ such that $\boldsymbol{x}_{1}, \cdots, \boldsymbol{x}_{g-1}$ are different from the points $\boldsymbol{a}_{1}, \cdots, \boldsymbol{a}_{m}$ and $\boldsymbol{b}_{1}, \cdots, \boldsymbol{b}_{m}$. Then, after suitable choice of paths of integration, we have 


$$
\sum_{k=1}^{m} \mathfrak{w}\left(\boldsymbol{a}_{k}\right)=\sum_{k=1}^{m} \mathfrak{w}\left(\boldsymbol{b}_{k}\right)
$$

and

$$
f(\boldsymbol{p})=\eta \prod_{k=1}^{m} \frac{\theta\left(\mathfrak{w}(\boldsymbol{p})+\sum_{\ell=1}^{g-1} \mathfrak{w}\left(\boldsymbol{x}_{\ell}\right)-\mathfrak{w}\left(\boldsymbol{a}_{k}\right)-\mathfrak{c}\right)}{\theta\left(\mathfrak{w}(\boldsymbol{p})+\sum_{\ell=1}^{g-1} \mathfrak{w}\left(\boldsymbol{x}_{\ell}\right)-\mathfrak{w}\left(\boldsymbol{b}_{k}\right)-\mathfrak{c}\right)} .
$$

Here, $\eta$ is a quantity independent of $p$, and $\mathfrak{c}$ is defined in (4.1.5).

Now, we apply (4.2.1) to a meromorphic function on $R$

$$
f(\boldsymbol{p})=1-x(\boldsymbol{p}), \quad \boldsymbol{p}=(x, y) .
$$

Let the points on $R$ which are over the points $x=0, z_{1}, \cdots, z_{s}, 1, \infty$ on the $x$-sphere be $\boldsymbol{q}_{0}, \boldsymbol{q}_{z_{1}}, \cdots, \boldsymbol{q}_{z_{s}}, \boldsymbol{q}_{1}, \boldsymbol{q}_{\infty}$ respectively. Then the zeros of $f(\boldsymbol{p})$ are $n$ points $\boldsymbol{q}_{1}, \cdots, \boldsymbol{q}_{1}$ and the poles of $f(\boldsymbol{p})$ are $n$ points $\boldsymbol{q}_{\infty}, \cdots, \boldsymbol{q}_{\infty}$. Therefore, by (4.2.1) we have

$$
f\left(\boldsymbol{q}_{0}\right)=\eta \prod^{n} \frac{\theta\left(\mathfrak{w}\left(\boldsymbol{q}_{0}\right)+\sum_{\ell=1}^{g-1} \mathfrak{w}\left(\boldsymbol{x}_{\ell}\right)-\mathfrak{w}\left(\boldsymbol{q}_{1}\right)-\mathfrak{c}\right)}{\theta\left(\mathfrak{w}\left(\boldsymbol{q}_{0}\right)+\sum_{\ell=1}^{g-1} \mathfrak{w}\left(\boldsymbol{x}_{\ell}\right)-\mathfrak{w}\left(\boldsymbol{q}_{\infty}\right)-\mathfrak{c}\right)}
$$

and

$$
f\left(\boldsymbol{q}_{z_{i}}\right)=\eta \prod^{n} \frac{\theta\left(\mathfrak{w}\left(\boldsymbol{q}_{z_{i}}\right)+\sum_{\ell=1}^{g-1} \mathfrak{w}\left(\boldsymbol{x}_{\ell}\right)-\mathfrak{w}\left(\boldsymbol{q}_{1}\right)-\mathfrak{c}\right)}{\theta\left(\mathfrak{w}\left(\boldsymbol{q}_{z_{i}}\right)+\sum_{\ell=1}^{g-1} \mathfrak{w}\left(\boldsymbol{x}_{\ell}\right)-\mathfrak{w}\left(\boldsymbol{q}_{\infty}\right)-\mathfrak{c}\right)} .
$$

Thus, we have the following formula.

$$
\begin{aligned}
1-z_{i}= & \prod_{n}^{n} \frac{\theta\left(\mathfrak{w}\left(\boldsymbol{q}_{z_{i}}\right)+\sum_{\ell=1}^{g-1} \mathfrak{w}\left(\boldsymbol{x}_{\ell}\right)-\mathfrak{w}\left(\boldsymbol{q}_{1}\right)-\mathfrak{c}\right)}{\theta\left(\mathfrak{w}\left(\boldsymbol{q}_{z_{i}}\right)+\sum_{\ell=1}^{g-1} \mathfrak{w}\left(\boldsymbol{x}_{\ell}\right)-\mathfrak{w}\left(\boldsymbol{q}_{\infty}\right)-\mathfrak{c}\right)} \\
& \cdot \prod^{n} \frac{\theta\left(\mathfrak{w}\left(\boldsymbol{q}_{0}\right)+\sum_{\ell=1}^{g-1} \mathfrak{w}\left(\boldsymbol{x}_{\ell}\right)-\mathfrak{w}\left(\boldsymbol{q}_{\infty}\right)-\mathfrak{c}\right)}{\theta\left(\mathfrak{w}\left(\boldsymbol{q}_{0}\right)+\sum_{\ell=1}^{g-1} \mathfrak{w}\left(\boldsymbol{x}_{\ell}\right)-\mathfrak{w}\left(\boldsymbol{q}_{1}\right)-\mathfrak{c}\right)} .
\end{aligned}
$$

Now, we know that there exist a basis of differentials

$$
p_{1}(\zeta, \lambda) d \zeta, \cdots, p_{g}(\zeta, \lambda) d \zeta
$$

and a canonical homology basis 


$$
A_{1}(\zeta, \lambda), \cdots, A_{g}(\zeta, \lambda) ; B_{1}(\zeta, \lambda), \cdots, B_{g}(\zeta, \lambda)
$$

such that

$$
\int_{\zeta}^{A_{i}(\zeta, \lambda)} p_{j}(\zeta, \lambda) d \zeta=\delta_{i j}, \quad \int_{\zeta}^{B_{i}(\zeta, \lambda)} p_{j}(\zeta, \lambda) d \zeta=z_{i j}(\lambda)
$$

Here $p_{j}(\zeta, \lambda)$ and $A_{j}(\zeta, \lambda), B_{j}(\zeta, \lambda)(1 \leqq j \leqq g)$ are holomorphic in $\lambda$ for every fixed $\zeta$ in a bounded Jordan domain $D(\lambda)$ [3].

We take (4.2.6) as the basis of differentials and take (4.2.7) as the canonical homology basis in (4.2.5). First, we see that $c$ is holomorphic in $\lambda$ by (4.1.5). Second, we have for $j(1 \leqq j \leqq g)$

$$
\begin{aligned}
& w_{j}\left(\boldsymbol{q}_{z_{i}}\right)-w_{j}\left(\boldsymbol{q}_{1}\right)=\int_{\boldsymbol{q}_{1}}^{\boldsymbol{q}_{z_{i}}} p_{j}(\zeta, \lambda) d \zeta, \\
& w_{j}\left(\boldsymbol{q}_{0}\right)-w_{j}\left(\boldsymbol{q}_{\infty}\right)=\int_{\boldsymbol{q}_{\infty}}^{\boldsymbol{q}_{0}} p_{j}(\zeta, \lambda) d \zeta .
\end{aligned}
$$

These are half periods along the closed curves which can be represented by linear combinations of $A_{1}(\zeta, \lambda), \cdots, B_{g}(\zeta, \lambda)$ with constant coefficients respectively in a neighborhood of $\lambda_{0}$. Therefore they are holomorphic in 2. Third, put ${ }^{t} \mathfrak{p}(\zeta, \lambda)=\left(p_{1}(\zeta, \lambda), \cdots, p_{g}(\zeta, \lambda)\right)$. Then, we can select a general divisor $D=x_{1}+\cdots+x_{g-1}$ such that the integral

$$
\mathfrak{w}\left(\boldsymbol{x}_{\ell}\right)=\int_{p_{0}}^{x_{\ell}} \mathfrak{p}(\zeta, \lambda) d \zeta \quad(1 \leqq \ell \leqq g-1)
$$

is holomorphic in a neighborhood of $\lambda_{0}$. Because we know that if $\tilde{D}=$ $\boldsymbol{x}_{1}+\cdots+\boldsymbol{x}_{g-1}+\boldsymbol{x}_{g}$ is general, then $D=\boldsymbol{x}_{1}+\cdots+\boldsymbol{x}_{g-1}$ is general. We assume that $x_{1}, \cdots, x_{g}$ are distinct $g$ points on the Riemann surface. A necessary and sufficient condition for $\tilde{D}$ to be general is that the determinant $\operatorname{det}\left(p_{i}\left(\zeta_{j}, \lambda\right)\right)$ does not vanish. Here, $\zeta_{1}, \cdots, \zeta_{g}$ are the coordinates of $\boldsymbol{x}_{1}, \cdots, \boldsymbol{x}_{g}$ in the domain $D(\lambda)$ respectively. Then, $\operatorname{det}\left(p_{i}\left(\zeta_{j}, \lambda\right)\right)$ is a holomorphic function in $\zeta_{1}, \cdots, \zeta_{g}, \lambda_{1}, \cdots, \lambda_{s}$. Here, $\lambda=\left(\lambda_{1}, \cdots, \lambda_{s}\right)$. Therefore, if this function does not vanish at $\chi_{0}=\left(\zeta_{1}^{(0)}, \ldots, \lambda_{s}^{(0)}\right)$, then it does not vanish at all points $\chi=\left(\zeta_{1}, \cdots, \lambda_{s}\right)$ in a neighborhood of $\chi_{0}$. Hence we get the assertion.

Summarizing, we obtain the following theorem.

(4.2.11) THEOREM. The single valued holomorphic functions which we have obtained in (3.3.7) can be expressed in the form (4.2.5). 
We may say (4.2.11) is an extension of the representation of the Lambda function by Theta constants.

The author wishes to thank Professor Kenkichi Kasahara for his kind suggestion in a part of 3.3 .

\section{REFERENCES}

[1] L. V. Ahlfors-L. Bers, Riemann's mapping theorem for variable metrics. Ann. of Math., vol. 72 (1960), 385-404.

[2] P. Appell, Fonctions hypergéométriques et hypersphériques. Gautier-Villars, 1926.

[ 3 ] L. Bers, Holomorphic differentials as functions of moduli, Bull. Amer. Math. Soc., vol. 67 (1961), 206-210.

[ 4 ] H. Grauert-R. Remmert, Komplexe Räume. Math. Ann. Bd. 136 (1958), 245-318.

[5] A. Kuribayashi, On analytic families of compact Riemann surfaces with non-trivial automorphisms. Nagoya Math. J., vol. 28 (1966), 119-165.

[6] A. Kuribayashi, Covering Riemann surfaces and Theta functions. Bull. Facul. Sci. \& Eng. Chuo Univ., vol. 15 (1972), 1-13.

[ 7 ] E. Picard, Traité d'analyse II, III, Gautier-Villars, 1925.

[ 8 ] R. Remmert, Holomorphe und meromorphe Abbildungen komplexer Räume. Math. Ann. Bd. 133 (1957), 328-370.

[ 9 ] G. Shimura, On analytic families of polarized abelian varieties. Ann. of Math., vol. 78 (1963), 149-192.

[10] G. Shimura, On purely transcendental fields of automorphic functions of several variables. Osaka J. Math., vol. 1 (1964), 1-14.

[11] C. L. Siegel, Ausgewählte Fragen der Funktionentheorie, II, Göttingen, 1954.

Department of Mathematics

Faculty of Science and Engineering

Chuo University 